\title{
A COMMUTATIVE BANACH ALGEBRA WHICH FACTORIZES BUT HAS NO APPROXIMATE UNITS
}

\author{
MICHAEL LEINERT
}

\begin{abstract}
ABSTRACr. It is well known that any Banach algebra having bounded approximate units factorizes. For some time it was not clear if, conversely, factorization implied the existence of bounded approximate units. This was disproved by Paschke [3], but the problem remained open for commutative Banach algebras. We give an example of a commutative semisimple Banach algebra which factorizes but has not even unbounded approximate units.
\end{abstract}

If $A$ is a commutative Banach algebra, we say that $A$ factorizes if any element $a \in A$ can be written $a=b c$ with $b, c \in A$. We say that $A$ has approximate units if for any $a \in A$ and $\varepsilon>0$ there is some $b \in A$ such that $\|a-b a\|<\varepsilon$.

Let $S$ be the semigroup (with pointwise addition) of all real sequences $b=\left\{b_{n}\right\}_{n \in \mathbf{N}}$ with $b_{n}>0$ for almost all $n \in \mathbf{N}$, and let $A=l^{1}(S)$ be the corresponding semigroup algebra. Let $a \in A$ be given, $a=\sum_{n \in N} \lambda_{n} \varepsilon_{f_{n}}$, where $\varepsilon_{f_{n}}$ denotes the Dirac measure on $S$ concentrated at $f_{n}=\left\{f_{n k}\right\}_{k \in \mathbf{N}}$. For each $m \in \mathbf{N}$ let $H_{m}=\left\{f_{n m} \mid 1 \leqslant n \leqslant m, f_{n m}>0\right\}$ and define

$$
h_{m}= \begin{cases}\frac{1}{2} \min H_{m}, & H_{m} \neq \varnothing, \\ 1, & H_{m}=\varnothing .\end{cases}
$$

The sequence $h=\left\{h_{m}\right\}_{m \in \mathbf{N}}$ is in $S$, and by construction we have $g_{n}=f_{n}$ $h \in S$ for all $n \in \mathbf{N}$. This implies

$$
a=\varepsilon_{h} \cdot\left(\sum_{n \in \mathbf{N}} \lambda_{n} \varepsilon_{g_{n}}\right),
$$

so $A$ factorizes.

For $g \in S$ and $a \in A$ we have $\left\|\varepsilon_{g} \cdot a-\varepsilon_{g}\right\| \geqslant 1$, since $g$ is not contained in the support of $\varepsilon_{g} \cdot a$. So $A$ has no approximate units. $A$ is semisimple, being a subalgebra of a commutative group algebra.

$\mathrm{I}$ am indebted to $\mathrm{H}$. Behncke for suggesting a simplification of the original proof.

\section{REFERENCES}

1. P. J. Cohen, Factorization in group algebras, Duke Math. J. 26(1959), 199-205. MR21 \#3729.

2. E. Hewitt and K. A. Ross, Abstract harmonic analysis. Vol. II: Structure and analysis for

Received by the editors January 27, 1975.

AMS (MOS) subject classifications (1970). Primary 43A20, 46J10.

Key words and phrases. Banach algebra, factorization, approximate units. 
compact groups. Analysis on locally compact Abelian groups, Die Grundlehren der math. Wissenschaften, Band 152, Springer-Verlag, Berlin and New York, 1970. MR41 \#7378; erratum, 42, p. 1825.

3. W. L. Paschke, A factorable Banach algebra without bounded approximate unit, Pacific J. Math. 46(1973), 249-251. MR48 \#2765.

FAKULTÄT FÜR MATHEMATIK, UNIVERSITÄT BIELEFELD, 48 BIELEFELD, POSTFACH 8640, WEST GERMANY 